\title{
Enhancing-ARDS diagnostics for ICU patients: a retrospective, nested case-control study to develop a biomarker-based model
}

\section{Xuan Fu}

Emergency Department, State Key Laboratory of Complex Severe and Rare Diseases, Peking Union Medical College Hospital, Chinese Academy of Medical Science and Peking Union Medical College Jinle Lin

The second school of Clinical Medicine, Southern Medical University

\section{Samuel Seery}

Department of Humanities, Peking Union Medical College, Chinese Academy of Medical Sciences and Peking Union Medical College

\section{Wuyuan Tao}

Department of Emergency and Critical Care Medicine, Affiliated Baoan Hospital of Shenzhen, The second school of Clinical Medicine, Southern Medical University

Yanxiu Lu

School of Medicine, Guangdong medical University, Shenzhen Jianbing Ye

Department of Emergency and Critical Care Medicine, Affiliated Baoan Hospital of Shenzhen, The second school of Clinical Medicine, Southern Medical University

\section{Shiyong Zeng}

Department of Emergency and Critical Care Medicine, Affiliated Baoan Hospital of Shenzhen, The second school of Clinical Medicine, Southern Medical University

\section{Jian Wei}

Department of Emergency and Critical Care Medicine, Affiliated Baoan Hospital of Shenzhen, The second school of Clinical Medicine, Southern Medical University

\section{Yi Luo}

Department of Emergency and Critical Care Medicine, Affiliated Baoan Hospital of Shenzhen, The second school of Clinical Medicine, Southern Medical University

\section{You Shang}

Department of Emergency and Critical Care Medicine, Affiliated Baoan Hospital of Shenzhen, The second school of Clinical Medicine, Southern Medical University

Wenwu Zhang ( $\nabla$ eccm_bah@foxmail.com)

Department of Emergency and Critical Care Medicine, Affiliated Baoan Hospital of Shenzhen, The second school of Clinical Medicine, Southern Medical University 


\section{Xuezhong Yu}

Emergency Department, State Key Laboratory of Complex Severe and Rare Diseases, Peking Union Medical College Hospital, Chinese Academy of Medical Science and Peking Union Medical College Jian Wu

Department of Respiratory, East Zone Sixth Division, Guangdong Provincial People's Hospital, Guangdong Academy of Medical Sciences, Guangdong Provincial Geriatrics Institute

\section{Jun Xu}

Emergency Department, State Key Laboratory of Complex Severe and Rare Diseases, Peking Union Medical College Hospital, Chinese Academy of Medical Science and Peking Union Medical College

\section{HuaDong Zhu}

Emergency Department, State Key Laboratory of Complex Severe and Rare Diseases, Peking Union Medical College Hospital, Chinese Academy of Medical Science and Peking Union Medical College

\section{Qingli Dou}

Department of Emergency and Critical Care Medicine, Affiliated Baoan Hospital of Shenzhen, The second school of Clinical Medicine, Southern Medical University

\section{Lijun Wang}

Department of Emergency and Critical Care Medicine, Affiliated Baoan Hospital of Shenzhen, The second school of Clinical Medicine, Southern Medical University

\section{Xiaobin Zeng}

Center Lab of Longhua Branch and Department of Infectious disease, Shenzhen People's Hospital, second Clinical Medical College of Jinan University

\section{Research Article}

Keywords: Acute respiratory distress syndrome, Biomarkers, Club cell protein 16, Angiopoietin 2, Soluble receptor for advanced glycation end-products, High-mobility group box 1 protein, Surfactant protein D, Intensive care unit

Posted Date: April 12th, 2021

DOl: https://doi.org/10.21203/rs.3.rs-400376/v1

License: (c) (i) This work is licensed under a Creative Commons Attribution 4.0 International License. Read Full License 


\section{Enhancing-ARDS diagnostics for ICU patients: a retrospective, nested case-control study to develop a biomarker-based model}

Xuan $\mathrm{Fu}^{1 \dagger}$, Jinle Lin ${ }^{2,3,4 \dagger}$, Samuel Seery ${ }^{5}$, Wuyuan $\mathrm{Tao}^{4}, \mathrm{Yanxiu} \mathrm{Lu}^{4,6}$, Jianbing $\mathrm{Ye}^{4}$, Shiyong Zeng $^{4}$, Jian $\mathrm{Wei}^{4}$, Yi Luo ${ }^{4}$, You Shang ${ }^{4}$,Wenwu Zhang ${ }^{4,6^{*}}$, Xuezhong $\mathrm{Yu}^{1 *}$, Jian $\mathrm{Wu}^{3,2^{*}}$, Jun $\mathrm{Xu}^{1}$, HuaDong Zhu ${ }^{1}$, Qingli Dou ${ }^{4,6}$, Lijun Wang ${ }^{4,6}$, Xiaobin Zeng ${ }^{7,8}$

Xuan Fu and Jinle Lin contribute equally to this work.

*co-correspondence:

Wenwu Zhang. E-mail: eccm_bah@foxmail.com;

Xuezhong Yu. E-mail: yxz@medmail.com.cn;

Jian Wu. Email: sywujian@scut.edu.cn.

\section{Author details}

${ }^{1}$ Emergency Department, State Key Laboratory of Complex Severe and Rare Diseases, Peking Union Medical College Hospital, Chinese Academy of Medical Science and Peking Union Medical College, Beijing, China.

${ }^{2}$ The second school of Clinical Medicine, Southern Medical University, Guangzhou, China.

${ }^{3}$ Department of Respiratory, East Zone Sixth Division, Guangdong Provincial People's Hospital, Guangdong Academy of Medical Sciences, Guangdong Provincial Geriatrics Institute, Guangzhou, China.

${ }^{4}$ Department of Emergency and Critical Care Medicine, Affiliated Baoan Hospital of Shenzhen, The second school of Clinical Medicine, Southern Medical University, Shenzhen, China.

${ }^{5}$ Department of Humanities, Peking Union Medical College, Chinese Academy of Medical Sciences and Peking Union Medical College, Beijing, China.

${ }^{6}$ School of Medicine, Guangdong medical University, Shenzhen, China. 
${ }^{7}$ Center Lab of Longhua Branch and Department of Infectious disease, Shenzhen People's Hospital, second Clinical Medical College of Jinan University, Shenzhen, China.

${ }^{8}$ Guangdong Provincial Key Laboratory of Regional Immunity and Diseases, Medicine School of Shenzhen University, Shenzhen, China.

Background: To investigate whether a series of biomarkers including club cell protein 16 (CC16), angiopoietin 2(Ang-2), soluble receptor for advanced glycation end-products (sRAGE), high-mobility group box 1 protein (HMGB1), and surfactant protein $\mathrm{D}$ (SPD) could be utilized for identifying patients, thereby increasing the diagnostic value of acute respiratory distress syndrome(ARDS) in intensive care unit (ICU).

Methods: 211 ICU admissions were enrolled in this retrospective, nested case-control study. These patients were then divided into ARDS $(n=79)$ and non-ARDS $(n=132)$ groups according to the Berlin criteria on ICU day 1. Patient characteristics, vital signs, and laboratory examinations were collected within three hours of admission. Five inflammatory associated plasma biomarkers, as well as lung epithelial and endothelial injury which included CC16, Ang-2, sRAGE, HMGB1 and SPD were measured in the morning of day two in the ICU. Diagnostic values were analyzed with receiver operating characteristic (ROC) curves. Pearson's product-moment correlation coefficient and multivariate logistic regression analysis were applied for predictive purposes.

Results: C-reactive protein (CRP), NT-proBNP, and PH values for traditional 
indicators and five biomarkers were analyzed with an objective ARDS indicator, the $\mathrm{PaO}_{2} / \mathrm{FiO}_{2}$ ratio. Evidence suggests that only four of potential indicators analyzed here, and CRP hold high diagnostic value. The area under curve (AUC) for each were as follows: CC16 (AUC: 0.752; 95\%CI0.680-0.824), Ang-2 (AUC: 0.695; 95\%CI 0.620 -0.770), HMGB1 (AUC: 0.668; 95\%CI 0.592-0.744), sRAGE (AUC: 0.665; 95\%CI 0.588-0.743), CRP (AUC: 0.701; 95\%CI 0.627-0.776). No single indicator surpassed the diagnostic capability of the $\mathrm{PaO}_{2} / \mathrm{FiO}_{2}$ ratio which had an AUC: $0.844(95 \%$ CI $0.789-0.898)$, especially in terms of sensitivity. However, when the binary logistic model was transformed and the model was built, the AUC increased from $0.647(95 \%$ CI $0.568-0.726)$ to $0.911(95 \%$ CI $0.864-0.946)$. Among the combinations tested, $\mathrm{PaO}_{2} / \mathrm{FiO}_{2}+\mathrm{CRP}+\mathrm{Ang}-2+\mathrm{CC} 16+\mathrm{HMGB} 1$ resulted in an $\mathrm{AUC}$ of 0.910 (95\%CI 0.863-0.945), while $\mathrm{PaO}_{2} / \mathrm{FiO}_{2}+\mathrm{CRP}+$ Ang-

2+CC16+HMGB1+sRAGE+SPD have an AUC of 0.911(95\%CI 0.864-0.946).

Conclusions: A combination of the assessed biomarkers could enhance ARDS diagnostics, which has obvious ramifications for patient care and prognosis. It may be possible to develop a predictive ARDS nomogram; however, of the combinations tested here, we would recommend $\mathrm{PaO}_{2} / \mathrm{FiO}_{2}+\mathrm{CRP}+\mathrm{Ang}-2+\mathrm{CC} 16+\mathrm{HMGB} 1$ for clinical practice. This is because of the cost implications in contrast with the benefit involved in utilizing the more elaborate model. Although, further health economics research is required to consider this opportunity cost for emergency care policy. Keywords: Acute respiratory distress syndrome; Biomarkers; Club cell protein 16; Angiopoietin 2; Soluble receptor for advanced glycation end-products; High-mobility 
group box 1 protein; Surfactant protein D; Intensive care unit

\section{Introduction}

Acute respiratory distress syndrome (ARDS) is a common disease characterized by permeability pulmonary edema and refractory hypoxemia in intensive care unit $(\mathrm{ICU})^{1}$. ARDS is associated with high morbidity and mortality rates although, it remains underdiagnosed and therefore all too often untreated ${ }^{2}$. The traditional diagnostic methods mentioned in the Berlin definition ${ }^{3}$, such as the ratio of partial pressure in arterial oxygen over the fraction of inspired oxygen $\left(\mathrm{PaO}_{2} / \mathrm{FiO}_{2}\right)$ and standard X-rays could perhaps be updated. The prevalence of ARDS and underdiagnosis with delays in identification of cases limits the effect of any intervention/s once administered. Physicians must have the most advanced diagnostics in order to improve clinical decision-making and at present this may not be the case for ARDS.

Biomarkers are indicators of pathophysiological processes, and prove insight into the biological responses to therapeutics. Biomarkers are increasingly becoming common place in both clinical research for participant selection but also for clinical practice such as triage. For example, biomarkers such as procalcitonin (PCT), Creactive protein (CRP), and interleukin-6 (IL-6) are frequently measured for sepsis, and Cardiac Troponin-I (cTnI) is now commonly used for acute myocardial infarction. These biomarkers provide timely information which can be effectively 
used to tailor therapeutic strategies around individual characteristics ${ }^{4,5}$. Therefore, having an appropriate biomarker (or combination of biomarkers) can help to determine disease characteristics as well as the sensitivity and specificity of an intervention/s.

To date, more than 20 biomarkers have proven useful for diagnosing or predicting ARDS $^{1}$. Fremont ${ }^{6}$ and Ware ${ }^{7}$ have also proposed that combining biomarkers will enhance ARDS diagnostics compared to a single biomarker. This is, because a specific combination is likely to improve both the accuracy and reliability of diagnosis and therefore prognostics. However, when developing biomarker-based models, it is necessary to avoid repeating measures for those with dissimilar pathophysiologies. Therefore, biomarkers derived through basic research into different pathophysiological pathways for inflammation, lung epithelial and endothelial injury were chosen for this study. These included club cell protein 16 (CC16), angiopoietin 2(Ang-2), soluble receptor for advanced glycation end-products (sRAGE), high-mobility group box 1 protein (HMGB1) and surfactant protein D $(\mathrm{SPD})^{2}$

\section{Methods}

\section{Study population}

ICU patients were enrolled between March 2013 and March 2017. The following eligibility criteria were necessary for inclusion: 1) patient age $>18$ and $<75$;2) 
expected ICU stay $>24 \mathrm{~h}$; 3 ) blood samples were collected $<6 \mathrm{~h}$ after admission; 4) diagnosis had been conformed prior to discharge. Those who did not meet all of the criteria were excluded. Written informed consent was then requested from potential participants or their legal representatives. The institutional human ethics committee of the affiliated Baoan Hospital of Shenzhen, Southern Medical University approved our study protocols (BYL 20141007). Research involving human participants, human material, or human data have been performed in accordance with the Declaration of Helsinki. All methods were carried out in accordance with relevant guidelines and regulations.

\section{Data collection and outcome of patients}

Values at baseline were recorded within $3 \mathrm{~h}$ of admission to the ICU, including individual characteristics (i.e., age, gender, comorbidities, and risk factors of ARDS), Acute Physiology and Chronic Health Evaluation II score (APACHE II), vital signs (i.e., blood pressure, body temperature, respiratory rate, and heart rate). Physiological variables for the $\mathrm{PaO}_{2} / \mathrm{FiO}_{2}$ ratio, as well as C-reactive protein (CRP), white blood cell count (WBC), the N-terminal of the prohormone brain natriuretic peptide (NT-proBNP), PH value, serum total protein (TP), D-Dimer, serum creatinine concentration (Scr), and lactic acid (Lac) were determined synchronously within $3 \mathrm{~h}$ of admission. Duration of mechanical ventilation (MV), mortality at Day-7, and Day-28 were recorded for all participants. 


\section{Diagnosis criteria and subgroups}

ARDS is diagnosed according to the Berlin definition ${ }^{3}$ which stipulates: 1)ARDS has an acute onset, of less than 7 days; with 2) bilateral opacity (consistent with pulmonary edema), as detected by CT or X-ray; and 3) $\mathrm{PaO}_{2} / \mathrm{FiO}_{2}$ ratio of less than $300 \mathrm{mmHg}$, with ventilation support (Positive End Expiratory Pressure or Continuous Positive Airway Pressure $\geqslant 5 \mathrm{mmH}_{2} \mathrm{O}$ ). Two senior physicians make a diagnosis based on patients' conditions within the first 48 hours of admission. All participants were divided into an ARDS or non-ARDS groups for further retrospective analysis.

\section{Measurement of serum CC16, Ang-2, sRAGE, HMGB1 and SPD}

Blood samples at Day-1 and Day-2 were separately collected from the radial artery within 3 hours and 24 hours of admission to ICU. Blood samples were then centrifuged at $3000 \mathrm{rpm}$ for $10 \mathrm{~min}$ and upper serum stored in EP tubes at an ultra-low temperature refrigerator $\left(-80^{\circ} \mathrm{C}\right)$ until required for analysis. Serum CC16, Ang-2, sRAGE, and SPD concentrations were determined using ELISA kits (R\&D Systems, Minneapolis, USA) and HMGB1 concentration were determined using ELISA kits (Elabscience Biotechnology Co., Ltd, Wuhan, Hubei, China), following the manufacturer's instructions. Each sample was measured in duplicate and assaying was conducted using the ELISA kits. The researchers who performed these analyses were 
blinded to group assignment.

\section{Statistical analysis}

Data are presented as the means with standard deviations or numbers (proportion) as indicated. Student's t-test was then performed to compare serum concentrations for Ang-2, CC16, sRAGE, SPD, and HMGB1 between the two groups, when distribution was considered to be normal. Conversely, when Guassian distribution was not evident, we adopted Mann-Whitney's U-test. Categorical data were compared using standard Chi-square tests. Correlations between Ang-2, CC16, sRAGE, SPD, HMGB1, $\mathrm{PaO}_{2} / \mathrm{FiO}_{2}, \mathrm{PH}, \mathrm{WBC}, \mathrm{CRP}$, NT-proBNP, TP, DDimer, albumin, and Scr were estimated using Pearson's linear regression coefficients.

Receiver operating characteristic (ROC) curves were utilized to assess the optimal area under the curves (AUC) with corresponding 95\% confidence intervals (CI). The optimal cut-off value, sensitivity, specificity, negative predictive value (NPV), and positive predictive value (PPV) were calculated after taking Youden's maximum (i.e., sensitivity+specificity-1). Statistical analysis was performed using R (v3.5.1, R Foundation for Statistical Computing, Vienna, Austria); A $p$-value $<0.05$ was considered the threshold for statistical significance. Graphs were created using GraphPad Prism software (v 3.0, GraphPad Software Inc., La Jolla, CA, USA) and MedCalc statistical software (v19.0.7, MedCalc Software Ltd, Ostend, 
Belgium). Corrplot was used to calculate correlation coefficient and to measure the significance of each correlation.

\section{Results}

\section{Participants and demographics}

Between $1^{\text {st }}$ March, 2013, and $1^{\text {st }}$ March, 2017, 1,121 ICU patients were initially considered eligible. Please see Fig. 1 for a complete flowchart of the process from screening to analysis. Those discharged within 24 hours $(n=253)$, those beyond the age range $(\mathrm{n}=198)$ and those who did not provide informed consent within the prespecified 12-hour window $(\mathrm{n}=78)$, were excluded. A further 381 potential candidates were excluded due to incomplete blood samples $(\mathrm{n}=198)$, incomplete clinical data ( $\mathrm{n}$ $=152)$ and discharge with unclear diagnosis $(n=31)$. A final cohort of 211 patients was recruited, of whom $37.4 \%(n=79)$ had been diagnosed with ARDS within the first day, and $62.6 \%(n=132)$ who did not meet the Berlin criteria (Fig.1). 


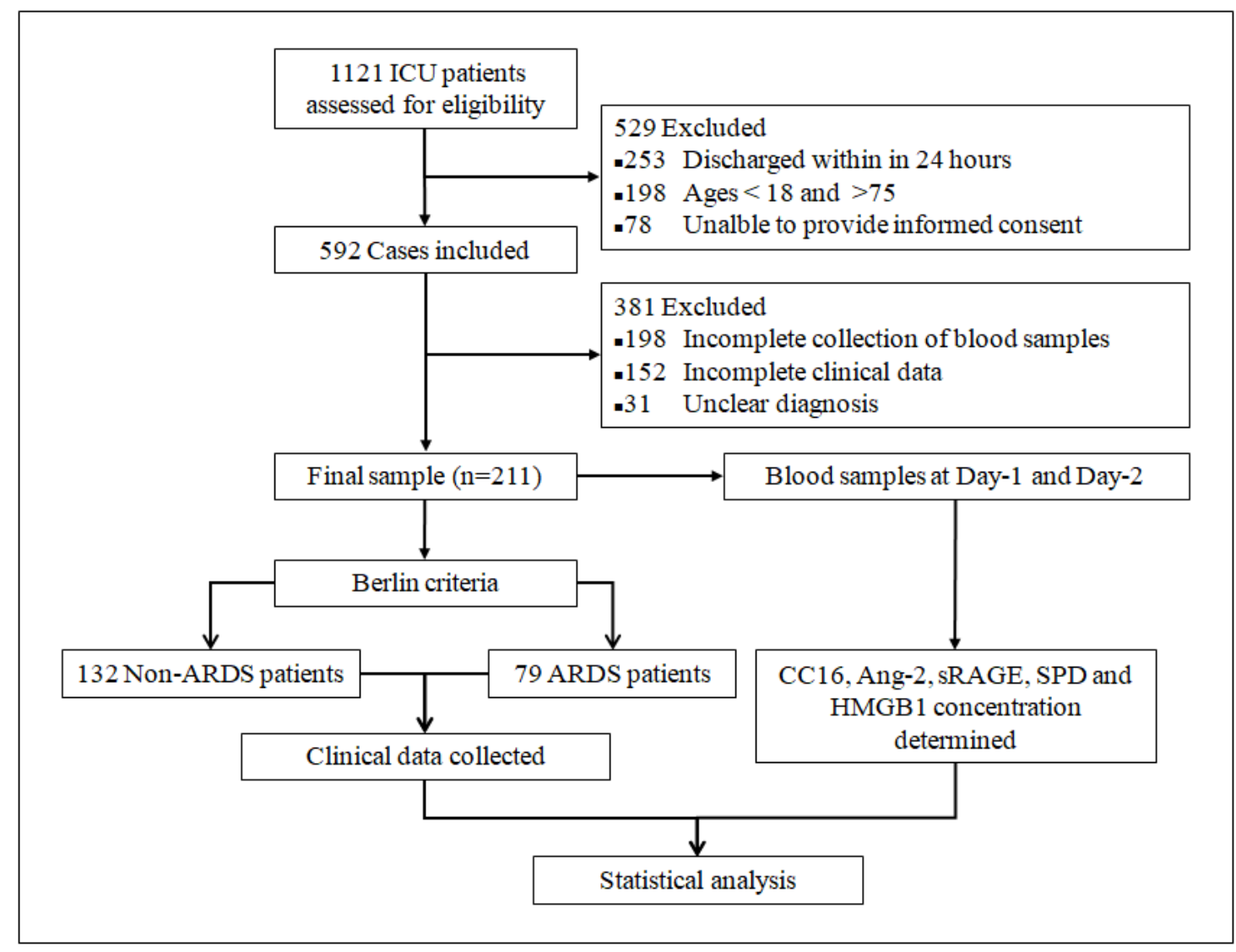

Fig.1 Flowchart of enrolment and study processes

Reasons for exclusion were not mutually exclusive or exhaustive because there may have been a number of reasons for exclusion.

Abbreviations: ICU, intensive care unit; ARDS, acute respiratory distress syndrome; $\mathrm{CC} 16$, club cell protein 16; Ang2, angiopoietin 2; sRAGE, soluble receptor for advanced glycation endproducts; SPD, surfactant protein D; HMGB1, high mobility group box 1

Demographics and clinical characteristics of two groups are presented in Table 1. The $p$ values provided are the result of comparisons between the ARDS and NonARDS groups using Student's t test, or Chi-square test. The median age was 54.68 $( \pm 18.94)$ years and $63.3 \%(50 / 79)$ were male in ARDS group. At baseline, patients in the ARDS group had higher CRP levels and lower $\mathrm{PaO}_{2} / \mathrm{FiO}_{2}$ ratios than patients 
in non-ARDS group. Comorbidities were similarly distributed in both groups.

Pneumonia had a higher rate in ARDS group although; no other major risk factors

were significantly different between the groups. APACHE II scores for the severity of illness, ventilation time, length of ICU stay and overall hospitalization were not significantly different between the two groups. However, 7-day mortality and 28-day mortality were higher in ARDS group.

Table 1. Demographics and clinical characteristics

\begin{tabular}{|c|c|c|c|}
\hline Variables & $\operatorname{ARDS}(n=79)$ & Non-ARDS(n=132) & Pvalue \\
\hline Age, years & $54.68 \pm 18.94$ & $49.79 \pm 17.05$ & 0.058 \\
\hline Male, n (\%) & $50(63.3 \%)$ & $81(61.4 \%)$ & 0.780 \\
\hline \multicolumn{4}{|l|}{ Comorbidities } \\
\hline Hypertension, n (\%) & $24(30.4 \%)$ & $41(31.1 \%)$ & 0.917 \\
\hline Diabetes, n (\%) & $17(21.6 \%)$ & $27(20.5 \%)$ & 0.854 \\
\hline Coronary heart disease, $\mathrm{n}(\%)$ & $11(13.9 \%)$ & $8(6.1 \%)$ & 0.053 \\
\hline Chronic lung disease, $\mathrm{n}(\%)$ & $1(1.8 \%)$ & $4(5.2 \%)$ & 0.446 \\
\hline Hepatitis B, n (\%) & $3(3.8 \%)$ & $3(2.3 \%)$ & 0.413 \\
\hline Chronic renal disease, $\mathrm{n}(\%)$ & $7(8.9 \%)$ & $13(9.8 \%)$ & 0.813 \\
\hline \multicolumn{4}{|l|}{ Risk Factors of ARDS } \\
\hline Pneumonia, n (\%) & $36(72.0 \%)$ & $25(34.7 \%)$ & $0.001^{*}$ \\
\hline Inhalation injury, $\mathrm{n}(\%)$ & $3(3.8 \%)$ & $0(0.0 \%)$ & 0.024 \\
\hline Pulmonary contusion, $\mathrm{n}(\%)$ & $1(1.3 \%)$ & $11(8.3 \%)$ & 0.032 \\
\hline Sepsis, n (\%) & $19(24.1 \%)$ & $19(14.4 \%)$ & 0.077 \\
\hline Cardiogenic shock, n (\%) & $1(1.3 \%)$ & $1(0.8 \%)$ & 0.712 \\
\hline Post-resuscitation, n (\%) & $8(10.1 \%)$ & $5(3.8 \%)$ & 0.064 \\
\hline Post-surgery, n (\%) & $24(30.4 \%)$ & $57(43.2 \%)$ & 0.064 \\
\hline Operative complication, $\mathrm{n}(\%)$ & $20(25.3 \%)$ & $22(16.7 \%)$ & 0.128 \\
\hline DIC, n (\%) & $5(6.3 \%)$ & $8(6.1 \%)$ & 0.937 \\
\hline Severe acute pancreatitis, $\mathrm{n}(\%)$ & $5(6.3 \%)$ & $7(5.3 \%)$ & 0.755 \\
\hline Poisoning, n (\%) & $2(2.5 \%)$ & $3(2.3 \%)$ & 0.905 \\
\hline Tuberculosis, n (\%) & $3(3.8 \%)$ & $3(2.3 \%)$ & 0.519 \\
\hline Pulmonary embolism, n (\%) & $1(1.3 \%)$ & $3(2.3 \%)$ & 0.604 \\
\hline \multicolumn{4}{|l|}{ Vital Signs } \\
\hline $\mathrm{T}\left({ }^{\circ} \mathrm{C}\right)$ & $36.99 \pm 1.03$ & $36.88 \pm 0.98$ & 0.704 \\
\hline HR, per min & $110.39 \pm 27.88$ & $101.64 \pm 27.21$ & 0.026 \\
\hline $\mathrm{RR}$, per min & $24.31 \pm 8.21$ & $23.18 \pm 7.17$ & 0.310 \\
\hline
\end{tabular}




\begin{tabular}{llll}
\hline $\mathrm{SBP}(\mathrm{mmHg})$ & $125.37 \pm 27.98$ & $127.25 \pm 24.97$ & 0.614 \\
\hline $\mathrm{DBP}(\mathrm{mmHg})$ & $76.73 \pm 17.69$ & $77.57 \pm 17.64$ & 0.741 \\
\hline $\mathrm{MAP}(\mathrm{mmHg})$ & $92.83 \pm 19.87$ & $94.07 \pm 18.48$ & 0.650 \\
\hline Physiological Variables & & & \\
\hline $\mathrm{PaO}_{2} / \mathrm{FiO}_{2}$ ratio & $180.64 \pm 99.12$ & $359.73 \pm 143.63$ & $0.001^{*}$ \\
\hline $\mathrm{CRP}(\mathrm{mg} / \mathrm{L})$ & $121.01 \pm 73.99$ & $67.76 \pm 63.79$ & $0.001^{*}$ \\
\hline $\mathrm{PH}$ value & $7.34 \pm 0.14$ & $7.38 \pm 0.12$ & 0.032 \\
\hline $\mathrm{Lac}(\mathrm{mmol} / \mathrm{L})$ & $4.87 \pm 8.24$ & $3.02 \pm 3.47$ & 0.061 \\
\hline $\mathrm{WBC}\left(\times 10^{9} / \mathrm{L}\right)$ & $14.37 \pm 8.57$ & $13.94 \pm 8.16$ & 0.717 \\
\hline $\mathrm{TP}(\mathrm{g} / \mathrm{L})$ & $57.93 \pm 14.20$ & $59.24 \pm 12.87$ & 0.493 \\
\hline $\mathrm{Alb}(\mathrm{g} / \mathrm{L})$ & $31.67 \pm 24.58$ & $31.95 \pm 7.53$ & 0.903 \\
\hline $\mathrm{D}-\mathrm{Dimer}(\mathrm{mg} / \mathrm{L})$ & $12.95 \pm 4.31$ & $5.53 \pm 9.94$ & 0.067 \\
\hline $\mathrm{Cr}(\mu \mathrm{mol} / \mathrm{L})$ & $164.40 \pm 289.79$ & $142.66 \pm 197.22$ & 0.520 \\
\hline NT-proBNP $(\mathrm{pg} / \mathrm{mL})$ & $712.17 \pm 924.59$ & $634.86 \pm 1055.74$ & 0.595 \\
\hline Evaluation index & & & \\
\hline APACHE II score & $19.40 \pm 6.88$ & $18.51 \pm 6.03$ & 0.573 \\
\hline Time of ventilation, days & $7.81 \pm 7.53$ & $6.56 \pm 9.67$ & 0.327 \\
\hline Length of ICU stay, days & $10.24 \pm 8.32$ & $10.84 \pm 11.26$ & 0.377 \\
\hline Length of hospitalization, days & $22.50 \pm 22.47$ & $29.08 \pm 63.68$ & 0.681 \\
\hline 7-day mortality, $\mathrm{n}(\%)$ & $18(22.8)$ & $10(7.6)$ & $0.002^{*}$ \\
\hline 28-day mortality, $\mathrm{n}(\%)$ & $30(38.0)$ & $23(17.4)$ & $0.001^{*}$ \\
\hline
\end{tabular}

Data shown as mean with corresponding standard deviations; $\mathrm{n}(\%)$;

${ }^{*}$ Statistically significant $p$ value.

Abbreviations: DIC, disseminated intravascular coagulation; APACHE II, Acute Physiology and Chronic Health Evaluation II; $\mathrm{PaO}_{2} / \mathrm{FiO}_{2}$, partial pressure of arterial oxygen to fraction of inspired oxygen

\section{Validation of a biomarker model for diagnosis of ARDS}

Levels of the five plasma biomarkers recorded from the entire cohort are shown in Table 2. Bar graphs were used to compare CC16, Ang-2, sRAGE, HMGB1 and SPD levels between the ARDS and non-ARDS groups. Of the five biomarkers, four significantly differed between groups under univariate analysis at ICU Day 1 and 
Day 2, i.e., CC16, Ang-2, sRAGE and HMGB1. See Figure 2 for confirmation.

Table 2. Serum levels of CC16, Ang-2, sRAGE, SPD and HMGB1 in ARDS groups and non-

ARDS groups at ICU Day 1 and Day 2

\begin{tabular}{lllcl}
\hline \multirow{2}{*}{ Biomarker } & \multicolumn{2}{c}{ Day 1 } & \multicolumn{2}{c}{ Day 2 } \\
\cline { 2 - 5 } & \multicolumn{1}{c}{ ARDS(n=79) } & non-ARDS $(\mathrm{n}=132)$ & \multicolumn{1}{c}{ ARDS(n=79) } & non-ARDS(n=132) \\
\hline CC16 $(\mathrm{ng} / \mathrm{mL})$ & $50.43 \pm 24.05^{*}$ & $29.30 \pm 20.06$ & $43.40 \pm 27.33^{*}$ & $28.98 \pm 21.38$ \\
Ang-2 $(\mathrm{pg} / \mathrm{mL})$ & $5597.20 \pm 3902.87^{*}$ & $3307.24 \pm 2477.35$ & $5405.13 \pm 3709.28^{*}$ & $3438.32 \pm 2650.91$ \\
sRAGE $(\mathrm{pg} / \mathrm{mL})$ & $2280.20 \pm 1946.72^{*}$ & $1371.95 \pm 1690.66$ & $2088.90 \pm 2364.73^{*}$ & $1400.18 \pm 2254.02$ \\
HMGB1 $(\mathrm{pg} / \mathrm{mL})$ & $2749.73 \pm 2128.15^{*}$ & $1606.92 \pm 1525.98$ & $2861.68 \pm 2148.54^{*}$ & $1595.75 \pm 1498.42$ \\
SPD $(\mathrm{ng} / \mathrm{mL})$ & $25.13 \pm 38.39^{*}$ & $15.51 \pm 19.35$ & $29.09 \pm 41.49$ & $19.92 \pm 26.31$ \\
\hline
\end{tabular}

* the difference between two groups was statistically significant
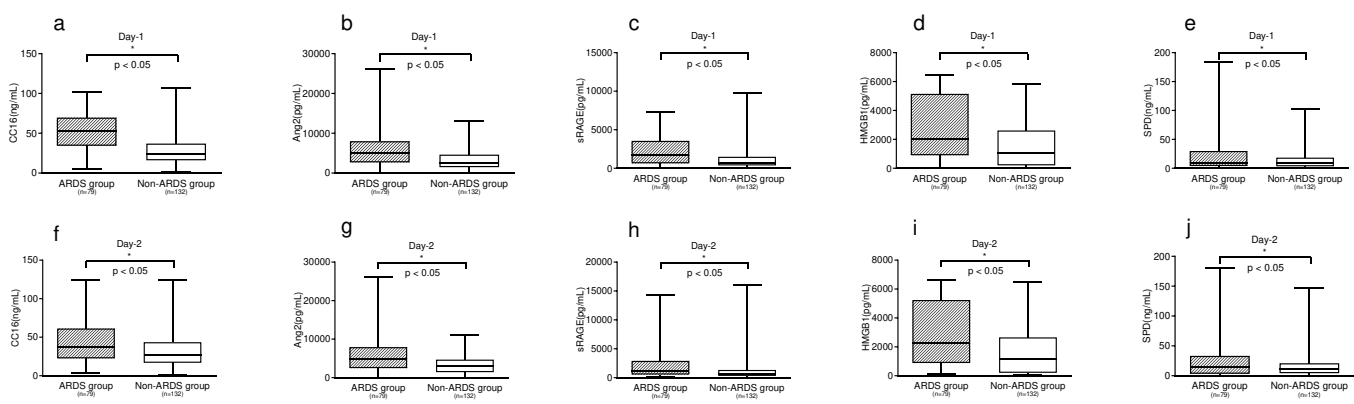

Fig. 2 Bar graph for comparison of CC16, Ang-2, sRAGE, HMGB1 and SPD levels between groups.

The $p$ values were derived from using student's t-test or Mann Whitney $\mathrm{U}$ test.

A logistic regression model was developed for ARDS diagnosis using all 5

biomarkers, and model performance was assessed using AUCs. As can be seen in

Table 3, the AUCs for each the biomarkers were as follows: CC16 0.752 (95\% CI

0.680-0.824), Ang-2 0.695 (95\% CI 0.620 - 0.770), HMGB1 0.668 (95\% CI 0.592-

0.744), sRAGE 0.665 (95\% CI 0.588- 0.743). 
Table 3. ROC analysis of Serum CC16, Ang-2, sRAGE, $\mathrm{HMGB} 1, \mathrm{SPD}, \mathrm{PaO}_{2} / \mathrm{FiO}_{2}$, CRP at diagnosing ARDS among critical care patients

\begin{tabular}{lcccccccc}
\hline Values & AUC & S. $\mathbf{E}$ & $\mathbf{9 5} \% \mathbf{C I}$ & Cut-off & Sensitivity & Specificity & NPV & PPV \\
\hline $\mathrm{CC} 16(\mathrm{ng} / \mathrm{ml})$ & $0.752^{\dagger}$ & 0.037 & $0.680,0.824$ & $>33.67$ & 77.2 & 69.7 & 83.62 & 60.39 \\
$\mathrm{Ang} 2(\mathrm{pg} / \mathrm{ml})$ & 0.695 & 0.038 & $0.620,0.770$ & $>3943.13$ & 62.0 & 69.7 & 75.39 & 55.04 \\
sRAGE $(\mathrm{pg} / \mathrm{ml})$ & 0.665 & 0.040 & $0.588,0.743$ & $>1283.83$ & 62.0 & 71.2 & 75.79 & 65.30 \\
$\mathrm{HMGB} 1(\mathrm{pg} / \mathrm{ml})$ & 0.668 & 0.039 & $0.592,0.744$ & $>823.9$ & 87.3 & 42.4 & 84.79 & 47.56 \\
$\mathrm{SPD}(\mathrm{ng} / \mathrm{ml})$ & 0.537 & 0.042 & $0.454,0.620$ & $>24.48$ & 31.6 & 83.3 & 67.05 & 53.10 \\
$\mathrm{PaO}_{2} / \mathrm{FiO}$ & $0.844^{\#}$ & 0.028 & $0.789,0.898$ & $<296.5$ & 93.7 & 69.7 & 94.86 & 64.92 \\
$\mathrm{CRP}(\mathrm{mg} / \mathrm{ml})$ & 0.701 & 0.038 & $0.627,0.776$ & $>88.0$ & 63.5 & 73.5 & 77.08 & 58.91 \\
\hline
\end{tabular}

Diagnostic values were assessed by the receiver operating characteristic curve (ROC). In the univariate logistic regression analyses, the diagnostic value of each factor was studied individually, introducing only one factor at a time into the model.

${ }^{\dagger}$ Among 5 biomarkers, CC16 provided the highest AUC of 0.752 with a sensitivity of $77.2 \%$ and a specificity of $69.7 \%$ at the optimal cut-off point of $33.67 \mathrm{ng} / \mathrm{mL}$ in patients with ARDS.

\# Among $\mathrm{PaO}_{2} / \mathrm{FiO}_{2}, \mathrm{CRP}$ and 5 biomarkers, $\mathrm{PaO}_{2} / \mathrm{FiO}_{2}$ provided the highest AUC of 0.844 with a sensitivity of $93.7 \%$ and a specificity of $69.7 \%$ at the optimal cut-off point of 296.5 in patients with ARDS.

Abbreviations: AUC, area under the curve; S.E, standard error; CI, confidence interval; NPV, negative predictive value; PPV, positive predictive value

\section{Correlations of biomarkers with traditional parameters and their diagnostic} value

As an objective indicator of $\mathrm{ARDS}, \mathrm{PaO}_{2} / \mathrm{FiO}_{2}$ resulted in the largest $\mathrm{AUC}$ of 
0.844 (95\% CI 0.789-0.898), as can be seen in Table 3. In Figure 3a, corrplot was used to plot the data series of $\mathrm{PaO}_{2} / \mathrm{FiO}_{2}$, five biomarkers, laboratory parameters and vital signs. CRP, NT-proBNP, PH value of traditional indicators and five biomarkers all correlated with $\mathrm{PaO}_{2} / \mathrm{FiO}_{2}$ although, only four (i.e., CC16, Ang-2, HMGB1, sRAGE) and CRP (AUC0.701, 95\% CI 0.627-0.776) had high diagnostic values, but no single marker was high as the $\mathrm{PaO}_{2} / \mathrm{FiO}_{2}$ ratio (please see table 3 for further analysis).

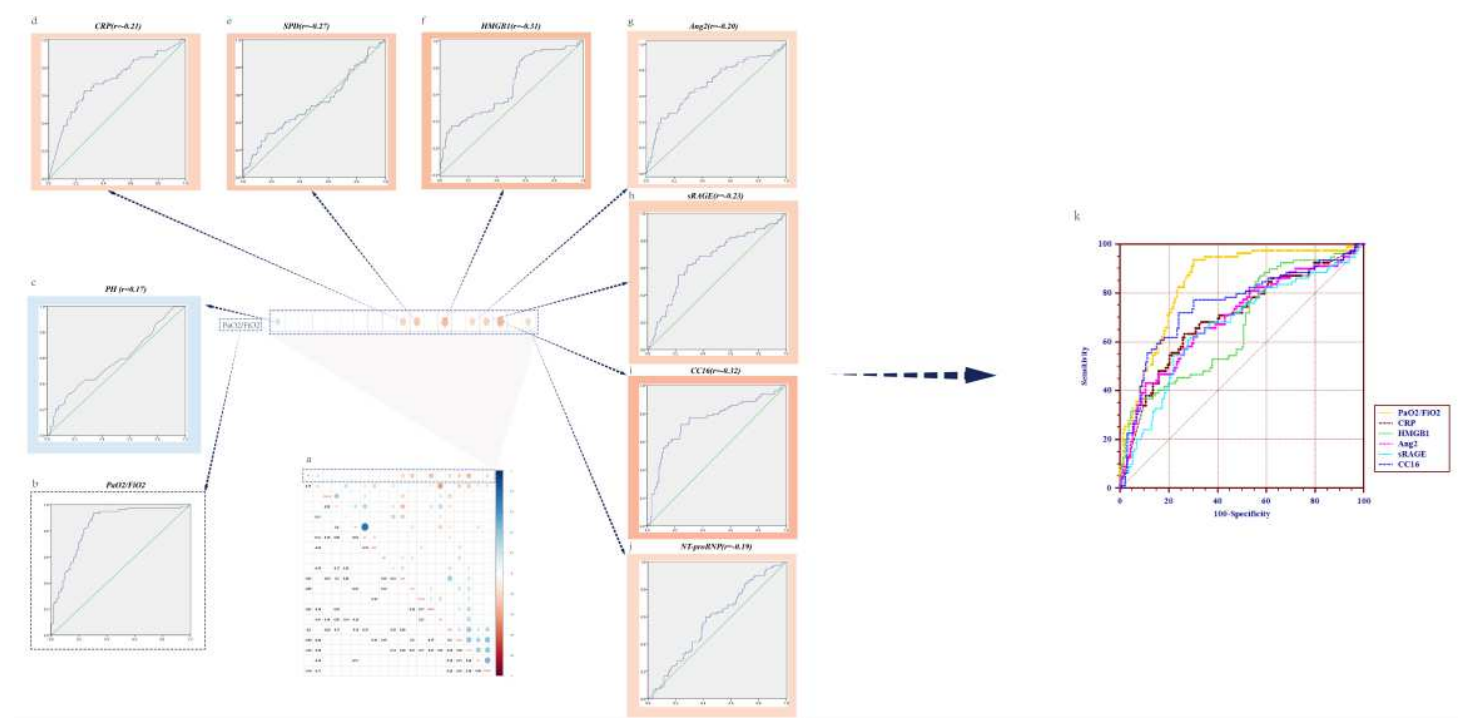

Fig. 3 (a) Correlations between P/F, PH, Albumin, TP, T, SP, DP, WBC, RR, H, CRP, SPD, Ddimer, HMGB1, Lac, Ang-2, sRAGE, CC16, Scr and NT-proBNP were investigated using Pearson's correlation coefficient. The ratio R was calculated by using Pearson's correlation coefficients ranging from -1 to 1 . Negative correlations have been highlighted in blue and positive correlations in red. Highlighted correlation coefficients indicate that correlations significantly differ from zero, and an emptyspace indicates the $\mathrm{r}$ ratio had no significant difference. (b-j) ROC curves of P/F, PH, CRP, SPD, HMGB1, Ang-2, sRAGE, CC16 and NT-proBNP in diagnosing ICU patients with ARDS. The Pearson's correlation between P/F and other indicators were referred to the $r$ value above. A negative value of $r$ indicated a negative correlation, or vice versa. (k) ROC 
curves analysis showed the AUC of P/F, CRP, HMGB1, Ang-2, sRAGE and CC16, respectively. A logistic regression model was fit for diagnosis performance of ARDS using P/F, CRP and four biomarkers; the model performance was assessed by the AUC of the ROC

Abbreviations: $\mathrm{P} / \mathrm{F}, \mathrm{PaO}_{2} / \mathrm{FiO}_{2}$; TP, serum total protein; $\mathrm{T}$, temperature; $\mathrm{SP}$, systolic blood pressure; DP, diastolic blood pressure; WBC, white blood cell count; RR, respiratory rate; $\mathrm{H}$, heart rate; $\mathrm{CRP}$, c-reactive protein; Lac, lactic acid; Scr, serum creatinine; $\mathrm{CC} 16$, club cell protein 16; Ang-2, angiopoietin 2; sRAGE, soluble receptor for advanced glycation end-products; HMGB1, high-mobility group box 1 protein; SPD, surfactant Protein D; NT-proBNP, the N-terminal of the prohormone brain natriuretic peptide; ICU, intensive care unit; ARDS, acute respiratory distress syndrome; AUC, area under the curve; ROC, receiver operator characteristic.

\section{Improvement of ROC value for the diagnosis of ARDS}

By translating from a binary logistic model, it was possible to build a model using various biomarker combinations (Table 4). As a result, the AUC increased from 0.647 (95\% CI 0.568-0.726) to 0.800 (95\% CI 0.737-0.863). With two biomarkers i.e., $\mathrm{CC} 16+\mathrm{Ang}-2$ providing the highest $\mathrm{AUC}$ of 0.787 with a sensitivity of $73.4 \%$ and a specificity of $77.3 \%$ in patients with ARDS. However, higher AUCs of 0.796 were provided in both CC16+Ang-2+HMGB1 and with the CC16+Ang-2+HMGB1+sRAGE combination. When combining five biomarkers, we observed the highest AUC value of 0.800 with a slightly lower sensitivity of $70.9 \%$ but a specificity of $79.5 \%$. 
Table 4. ROC analysis of combining CC16, Ang-2, sRAGE, HMGB1 and SPD at diagnosing

ARDS with different combination methods among critical care patients

\begin{tabular}{|c|c|c|c|c|c|c|c|}
\hline Value & AUC & SE & $95 \% \mathrm{CI}$ & Sensitivity & Specificity & NPV & PPV \\
\hline CC16+Ang-2 & $0.787^{*}$ & 0.034 & $0.721,0.854$ & 73.4 & 77.3 & 82.92 & 65.92 \\
\hline CC16+sRAGE & 0.754 & 0.036 & $0.682,0.825$ & 73.4 & 74.2 & 82.33 & 62.99 \\
\hline CC16+HMGB1 & 0.765 & 0.035 & $0.696,0.833$ & 67.1 & 77.3 & 79.69 & 63.88 \\
\hline CC16+SPD & 0.761 & 0.035 & $0.692,0.830$ & 74.7 & 72.0 & 82.62 & 61.48 \\
\hline Ang-2+sRAGE & 0.723 & 0.036 & $0.653,0.794$ & 81.0 & 50.8 & 81.71 & 49.62 \\
\hline Ang-2+HMGB1 & 0.742 & 0.036 & $0.670,0.813$ & 67.1 & 72.0 & 78.52 & 58.91 \\
\hline Ang-2+SPD & 0.724 & 0.037 & $0.650,0.797$ & 63.3 & 75.8 & 77.53 & 61.01 \\
\hline sRAGE+HMGB1 & 0.696 & 0.037 & $0.623,0.769$ & 57.0 & 73.5 & 74.06 & 56.27 \\
\hline sRAGE+SPD & 0.647 & 0.04 & $0.568,0.726$ & 63.3 & 65.9 & 75.00 & 52.62 \\
\hline HMGB1+SPD & 0.670 & 0.039 & $0.593,0.747$ & 43.3 & 87.1 & 71.96 & 66.76 \\
\hline CC16+Ang-2+sRAGE & 0.787 & 0.034 & $0.721,0.854$ & 74.7 & 75.8 & 83.35 & 64.87 \\
\hline CC16+Ang-2+HMGB1 & $0.796^{\dagger}$ & 0.033 & $0.732,0.860$ & 74.7 & 72.0 & 82.62 & 61.48 \\
\hline CC16+Ang-2+SPD & 0.794 & 0.033 & $0.730,0.858$ & 78.5 & 68.9 & 84.26 & 60.16 \\
\hline Ang-2+sRAGE+HMGB1 & 0.754 & 0.035 & $0.685,0.823$ & 74.7 & 66.7 & 81.49 & 57.31 \\
\hline Ang-2+sRAGE+SPD & 0.740 & 0.036 & $0.670,0.809$ & 73.4 & 65.9 & 80.54 & 56.29 \\
\hline sRAGE+HMGB1+SPD & 0.695 & 0.038 & $0.620,0.769$ & 58.2 & 77.3 & 75.55 & 60.54 \\
\hline $\begin{array}{l}\text { CC16+Ang-2+ } \\
\text { HMGB1+sRAGE }\end{array}$ & $0.796^{\&}$ & 0.033 & $0.732,0.860$ & 62.0 & 85.6 & 79.00 & 72.04 \\
\hline $\begin{array}{l}\text { CC16+Ang-2+ } \\
\text { sRAGE+SPD }\end{array}$ & 0.794 & 0.033 & $0.730,0.858$ & 75.9 & 72.5 & 83.40 & 62.28 \\
\hline $\begin{array}{l}\text { Ang-2+sRAGE+ } \\
\text { HMGB1+SPD }\end{array}$ & 0.764 & 0.034 & $0.696,0.831$ & 79.7 & 60.6 & 83.30 & 54.76 \\
\hline $\begin{array}{l}\text { CC16+Ang-2+sRAGE+ } \\
\text { HMGB1+SPD }\end{array}$ & $0.800^{\#}$ & 0.032 & $0.737,0.863$ & 70.9 & 79.5 & 82.03 & 67.42 \\
\hline
\end{tabular}

${ }^{*} \mathrm{CC} 16+\mathrm{Ang} 2$ showed the highest AUC of 0.787 with a sensitivity of $73.4 \%$ and a specificity of

$77.3 \%$ using two joint biomarkers.

CC16+Ang2+HMGB1 showed the highest AUC of 0.796 with a sensitivity of $74.7 \%$ and a specificity of $72 \%$ using three biomarkers combination.

${ }^{\&} \mathrm{CC} 16+$ Ang2+sRAGE+HMGB1+SPD showed the highest AUC of 0.796 with a sensitivity of $62 \%$ and a specificity of $85.6 \%$ using four biomarkers combination. 
\# Combination of all 5 biomarkers showed the AUC of 0.800 with a sensitivity of $70.9 \%$ and a specificity of $79.5 \%$.

The $\mathrm{PaO}_{2} / \mathrm{FiO}_{2}$ ratio is the traditional indicator for the diagnosis of ARDS, whereas CRP is an index that is relatively easy to obtain. This evidence also confirms that CRP has a significant influence in the diagnosis of ARDS (Table 3), despite negatively correlating with $\mathrm{PaO}_{2} / \mathrm{FiO}_{2}$ (Figure 3). We tried to combine $\mathrm{PaO}_{2} / \mathrm{FiO}_{2}$, CRP, and panels of biomarkers (Table 5) which garnered higher diagnostic significance. The $\mathrm{PaO}_{2} / \mathrm{FiO}_{2}+\mathrm{CRP}+\mathrm{Ang}-2+\mathrm{CC} 16+\mathrm{HMGB} 1$ combinations yielded an AUC of 0.910 (95\% CI 0.863-0.945), whereas the $\mathrm{PaO}_{2} / \mathrm{FiO}_{2}+\mathrm{CRP}+\mathrm{Ang}-2+\mathrm{CC} 16+\mathrm{HMGB} 1+\mathrm{sRAGE}+\mathrm{SPD}$ had the highest an AUC of $0.911(95 \%$ CI $0.864-0.946)$.

Table 5. ROC analysis of combining CC16, Ang-2, sRAGE, HMGB1 and SPD at diagnosing ARDS with different combination methods among critical care patients

\begin{tabular}{|c|c|c|c|c|c|c|c|}
\hline Value & $\mathbf{A U C}$ & SE & $95 \% \mathrm{CI}$ & Sensitivity & Specificity & NPV & PPV \\
\hline $\mathrm{PaO}_{2} / \mathrm{FiO}_{2}$ & 0.844 & 0.028 & $0.789,0.898$ & 93.7 & 69.7 & 69.7 & 94.8 \\
\hline CRP (mg/ml) & 0.701 & 0.038 & $0.627,0.776$ & 63.5 & 73.5 & 77.08 & 58.91 \\
\hline $\begin{array}{l}\text { CC16+Ang-2+sRAGE } \\
+ \text { HMGB1+SPD }\end{array}$ & 0.800 & 0.032 & $0.737,0.863$ & 70.9 & 79.5 & 67.4 & 82.03 \\
\hline $\begin{array}{l}\mathrm{PaO}_{2} / \mathrm{FiO}_{2}+\mathrm{CRP}+\mathrm{Ang}-2 \\
+\mathrm{CC} 16+\mathrm{HMGB} 1\end{array}$ & $0.910^{*}$ & 0.0207 & $0.863,0.945$ & 87.3 & 84.1 & 76.66 & 91.71 \\
\hline $\begin{array}{l}\mathrm{PaO}_{2} / \mathrm{FiO}_{2}+\mathrm{CRP}+\mathrm{Ang}-2 \\
+\mathrm{CC} 16+\mathrm{HMGB} 1 \\
+ \text { sRAGE+SPD }\end{array}$ & $0.911^{\#}$ & 0.0206 & $0.864,0.946$ & 86.1 & 83.3 & 75.5 & 90.9 \\
\hline
\end{tabular}

\# The highest AUC was 0.911 with a sensitivity of $86.1 \%$ and a specificity of $83.3 \%$ when combined all five biomarkers with $\mathrm{PaO}_{2} / \mathrm{FiO}_{2}$ and CRP. 
${ }^{*}$ The combination of $\mathrm{PaO}_{2} / \mathrm{FiO}_{2}, \mathrm{CRP}$, Ang-2, $\mathrm{CC} 16$ and HMGB1 showed an AUC of 0.910 with a sensitivity of $87.3 \%$ and a specificity of $84.1 \%$.

\section{Relationship of biomarkers with outcomes}

Across the entire ICU sample, higher serum levels of CC16 (Fig.4a to Fig.4d) and Ang-2 (Fig.4e to Fig.4h) were found in non-survivors. Consistently, CC16 at Day1(r=0.189, Fig.5a), Ang-2 at Day-1 ( $r=0.158$, Fig. 5b) and Day-2 (r=0.143, Fig. 5c) showed positive correlation with MV duration in the entire ICU patients. However, only $\mathrm{CC} 16$ on day 1 and day 2 showed higher mortality and statistical significance in 7-day and 28-day mortality in ARDS group. See Figure 4i to 41 for further details.

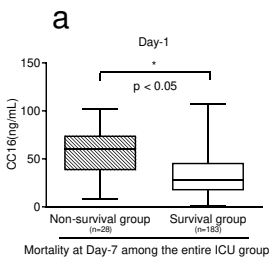

e
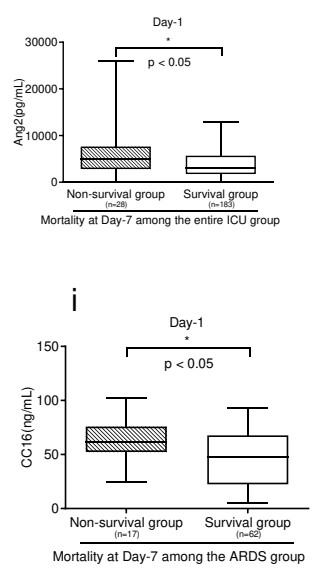

b

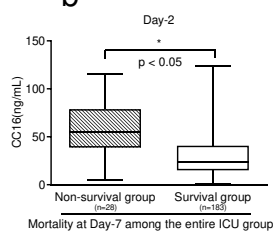

$f$
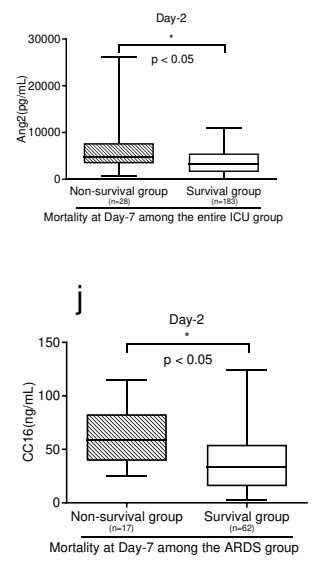

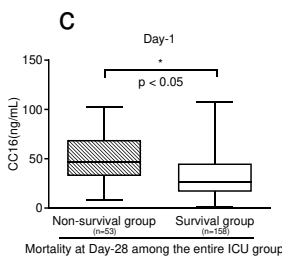

9
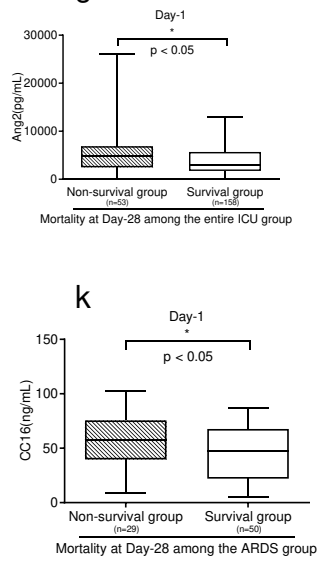

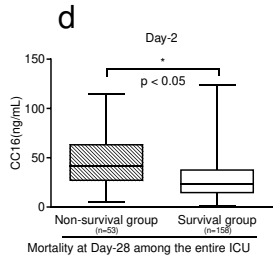

$\mathrm{h}$
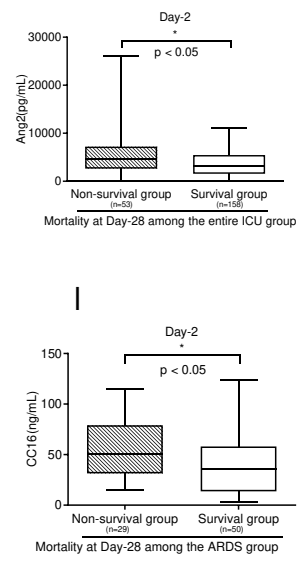

Fig. 4(a-h) Comparative bar charts of CC16 and Ang-2 between non-survival groups and survival groups across entire cohort. (i-j) Comparative bar charts of $\mathrm{CC} 16$ between non-survival groups 
and survival groups in ARDS only patients.

$p$ values were generated using Student's T-test.
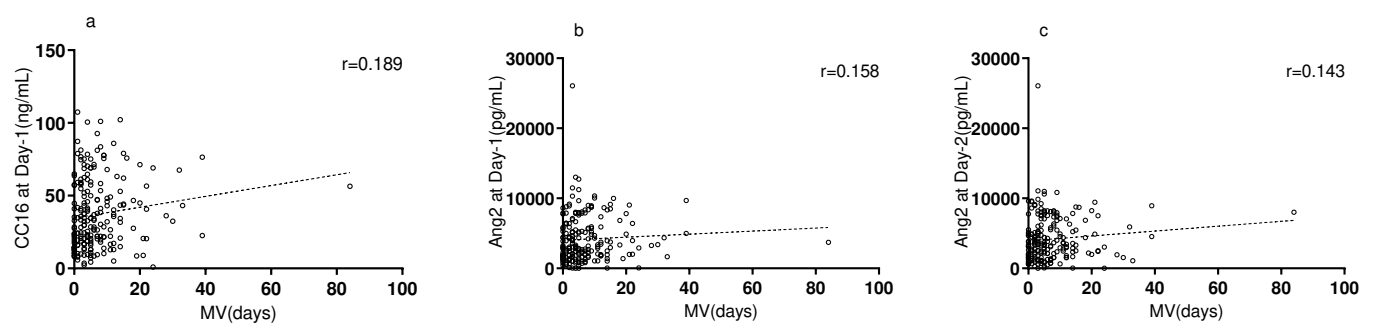

Fig.5 Scatter plots of a) the relationship between CC16 levels at Day-1 and duration of mechanical ventilation; b) the relationship between Ang-2 levels at Day-1 and duration of mechanical ventilation; c) the relationship between Ang-2 levels at Day-2 and duration of mechanical ventilation.

$r$ values were accounted from using Pearson correlation coefficient.

Abbreviations: MV, mechanical ventilation duration; r, correlation coefficient

\section{Discussion}

The primary objective was to consider whether intercalating series of biomarkers into traditional ARDS diagnostics would increase diagnostic accuracy for critically ill patients. We initially sought to confirm whether biomarkers such as CC16, Ang2, HMGB1 and sRAGE would prove useful for patients with ARDS. We then began to analyze potential correlations between theafore mentioned biomarkers and traditional indicators. We found that these biomarkers can supplement routine 
indicator such as the $\mathrm{PaO}_{2} / \mathrm{FiO}_{2}$ ratio and could prove useful in clinical practice. We also found that CC16, Ang-2 and HMGB1 detection when combined with $\mathrm{PaO}_{2} / \mathrm{FiO}_{2}$ and CRP can improve the diagnostic accuracy of traditional methods. Our evidence also suggests that $\mathrm{CC} 16$ and Ang-2 may prove useful for assessing lung function recovery, which positively correlates with mechanical ventilation time. Further, we speculate that CC16 might be useful as a prognostic indicator for ARDS patients who have higher comparative 7-day and 28-day mortality.

The relatively new Berlin definition made a number of improvements over previous definitions of acute lung injury as opposed to acute respiratory distress syndrome. As such, the newer Berlin definition removing acute lung injury and other ancillary indicators, categorizing ARDS into three levels of severity, which actually lowers the diagnostic criteria for ARDS. People have reason to doubt whether a large number of patients without diffuse alveolar damage pathological changes will be diagnosed as ARDS according to the Berlin definition, thereby increasing the incidence of ARDS and the cost of treatment. While according to the LUNG SAFE study, this increase ARDS prevalence which actually appears necessary because only $51.3-78.5 \%$ of ARDS cases are identified in clinical practice ${ }^{8}$. As mentioned within the introduction, one of the greatest problems is underdiagnose which could mean there is a lack of clinical awareness. Although, the main reason appears to be the lack of simple, accurate diagnostic tests, which leads to an over-reliance on consensus definitions. This necessitates the development of more objective measures for ARDS to ensure patients are treated more appropriately and in a timely manner. 
Researchers have suggested ${ }^{9}$ it necessary to establish a set of biomarkers in order to further develop specific medicaments and tailor interventions to individual needs. Evidence also demonstrates that clinically established biomarkers, such as white blood cell count, blood creatinine, inflammation indicators, etc., have less diagnostic value than plasma protein-based biomarkers. Therefore, plasma protein biomarkers are more likely to provide insight into the pathophysiology of ARDS and indeed into the distinct levels of severity as described in the newest Berlin definition. Fortunately, number of biomarkers identified through different pathophysiological pathways of inflammation, lung epithelial and endothelial injury, have been studied and have been found to be relevant in the diagnosis of ARDS $^{1}$. However, ARDS is not a single disease, but rather a syndrome with complex etiology, pathogenesis, pathophysiology and therefore testing may lack specificity.

There are however a wide array of biomarkers which show promise and some studies have investigated the potential of combining several biomarkers into ARDS diagnostics. For example, Fremont et al. ${ }^{6}$ found seven biomarkers including RAGE, PCPIII, BNP, Ang-2, IL-10, TNF- $\alpha$ and IL-8 had high diagnostic accuracy for ARDS diagnosis. Likewise, Ware et al. ${ }^{7}$ also tested a panel of biomarkers including vWF, SPD, TNFR, IL-6, IL-8, ICAM-1, PROTC and PAI-1, and found that combinations enhanced prognostic values compared to any single biomarker. More recently, Ware et al. ${ }^{10}$ studied a different set of biomarkers which included SPD, RAGE, IL-6, IL-8 and CC16, and found that this had a higher AUC for ARDS 
diagnosis. Although, one could argue due to sheer number of plasma protein-based biomarkers, we need a more systematic process for inclusion and exclusion based upon previous evidence. Additionally, there is evidence which suggests there are genetics in terms of thrombomodulin and endothelial protein $\mathrm{C}$ receptors which are associated with ARDS mortality ${ }^{11}$. This makes it necessary to investigate biomarkers across different ehicities because they are associated with dyregulated coagulation and therefore ARDS outcomes.

Through previous investigation ${ }^{12}$, we found that $\mathrm{CC} 16$ could be applied as an efficacious diagnostic biomarker for ARDS. In the present study, we attempted to add to this knowledge base by adding other biomarkers which have proven promising elsewhere. The hope was to extend the detection range and avoid repeat determinations for those with dissimilar pathophysiologies. As such, CC-16, sRAGE and SPD, which are associated with epithelial lung damage, were derived on an individual basis from club cells, type I and II alveolar epithelial cells. Likewise, Ang2 wasderived from individual endothelial cells and represent endothelial lung damage. HMGB1 were recorded from macrophages which relate to inflammatory changes. When the immune system is infected, inflammatory biomarkers change, including HMGB $1^{13}$, CRP and PCT change. Similarly, lung biomarkers including CC16 ${ }^{14}$, Ang$2^{15}, \mathrm{sRAGE}^{16}$ and $\mathrm{SPD}^{17}$ which are generally low in healthy individuals, are found to elevate in patients with a severely compromised blood gas barrier. There is evidence throughmouse modelling studies, that this complement of biomarkers synchronously increase $^{18}$. However, this is the first study to observe this in the development of 
ARDS. We also found that the AUC values increased with varying degrees after adding various biomarkers. This is consistent with the results of the study by Ware et $\mathrm{al}^{6,10}$, although the complement of biomarkers involved in this study is not the same.

At present, the $\mathrm{PaO}_{2} / \mathrm{FiO}_{2}$ ratio is the gold standard in ARDS diagnostics; however, the diagnostic performance is not ideal andit has limitation when considering other cardiac factors, as shown by the negative correlation with NTproBNP. Consequently, we theorized that the observed improvement was related to a reduction in the influence of cardiogenic factors. In the past, biomarker research in this field has predominantly focused on how to distinguish severe ARDS patients from cardiogenic pulmonary edema, with no biomarker being identified as capable of replacing the $\mathrm{PaO}_{2} / \mathrm{FiO}_{2}$ ratio in terms of sensitivity. We found that CRP not only has diagnostic value, but also negative correlates with $\mathrm{PaO}_{2} / \mathrm{FiO}_{2}$. Therefore, we iteratively tested various combinations of biomarkers with $\mathrm{CRP}$ and $\mathrm{PaO}_{2} / \mathrm{FiO}_{2}$, and found little difference between three and five in diagnostic sensitivity and specificity. Although of course, this means we are able to recommend the Ang-2, CC16, HMGB1, CRP and $\mathrm{PaO}_{2} / \mathrm{FiO}_{2}$ for clinical practice in order to save medical resources.

We also found that there are physiological interrelations between some of the investigated biomarkers and traditional indicators. Evidence from our previous research ${ }^{19}$ and other ${ }^{20,21}$ suggests that CC16 positively correlates with Scr, Lac and NT-proBNP. This might related to the anti-inflammatory function, immune system 
activation, and lactate metabolism pathway related to prephosphorylation. Likewise, the relationship between Ang-2 and albumin, and CRP, is partly consistent with previous results ${ }^{22}$, most of which were obtained in clinical samples. It was found that the pro-inflammatory properties may affect the leukocyte adhesion mechanism in inflammation. The association between sRAGE and NT-proBNP we observed might be also affected by cardiac remodeling and anti-inflammatory effects. Both BNP and NT-pro-BNP levels elevate in patients with chronic renal insufficiency, which are closely related to left ventricular hypertrophy and abnormal systolic function. Therefore, both have the potential to predict heart failure and mortality. This hypothesis seems to be supported by the results obtained in other types of patients ${ }^{23-26}$, where elevated levels of sRAGE were considered a sign of worsening cardiac function and mortality.

From a prognostic perspective, the association between serum Ang-2 and mortality is consistent with previous findings in patients with sepsis ${ }^{27,28}$. Consistently, multivariate regression analysis has shown that non-survivors are more likely to have biological dysfunction when admitted, which may help integrate biomarker-based predictive models to support clinicians assessing critically ill patients before confirming diagnosis. SPD appears to have diagnostic value for ARDS and shows superiority in prognostic value of interstitial lung disease ${ }^{29}$. However, previous research has found that the level of SPD is not related to lung contusion associated with $\mathrm{ARDS}^{30}$. In that study, the researchers also found that serum CC16 level is related to the volume of lung contusion and may not be affected by the overall 
severity of injury, age, gender or ventilation. This supports our findings that SPD should not be used alone to diagnose and evaluate the prognosis of ARDS. However, we found that the CC16 levels on Day-1 and Day-2 correlated with 7-day and 28-day mortality. Based on the pathophysiology of ARDS, prognosis appears to be reflected in CC16 levels which we suggest related to the blood gas barrier repair process. In our previous study ${ }^{31}$, we discovered the prognostic value of CC16 for non-invasive ventilation in critically ill patients. The findings of this study elaborate on this; suggesting that serum CC16 and Ang-2 in critically ill patients are closely related to the duration of mechanical ventilation. However, this is a tentative notion because subsequent lung infections such as hospital-acquired pneumonia, or ventilator-associated pneumonia, may have influenced our findings.

\section{Strength and limitations}

To the best of our knowledge, this is one of the few studies which combine plasma-based biomarkers and traditional indicators to evaluate ARDS in more than 200 patients $^{32,33}$. We also think by recruiting various critically ill patients, our research can be repeated at the local emergency and critical care center. The recommended combination could help clinicians identify ARDS in critically ill people although the findings will need to be externally validated across a larger Chinese population.

The patients enrolled in this study were also pretreated, prior to receiving ICU 
treatment. This of course creates, inconsistencies across an otherwise genetically population. Future research might try to develop a nomogram for this population although effort should be taken to reduce selection bias, where possible. There are a number of prospective advantages in intercalating plasma-based biomarkers, yet remain difficult to adopt these in clinical practice without commercially produced instanteous testings. Given this, we were only able to perform testing at the early stage of patient admission, not throughout the entire period of hospitalization.

However, we hope this evidence adds to a necessary, growing body of evidence and will help those considering the design of large-scale, prospective clinical studies in this field.

\section{Conclusion}

We investigated the detection of serum CC16, Ang-2, sRAGE, SPD and HMGB1 to identify patients with ARDS in those considered to be critically ill. Using an iterative approach to developing a combined detection method, we found that Ang2+CC16 $+\mathrm{HMGB} 1+\mathrm{CRP}+\mathrm{PaO}_{2} / \mathrm{FiO}_{2}$ enhances ARDS diagnostics substantially. However, Ang-2+CC16+HMGB1+CRP $+\mathrm{PaO}_{2} / \mathrm{FiO}_{2}$ plus sRAGE $+\mathrm{SPD}$ was superior. We also found that CC16 and Ang-2 might effect mortality and mechanical ventilation time. Further research into the underlying mechanisms is currently underway, which we hope will improve our understanding of ARDS physiology. We would also suggest there may be a need to develop a predictive nomogram as well as for health 
economics research to consider the trade-offs between the more basic model

recommended here and the more sophisticated model, which is marginally more accurate.

\section{Declarations}

\section{Ethics approval and consent to participate}

Research involving human participants, human material, or human data have been performed in accordance with the Declaration of Helsinki. All methods were carried out in accordance with relevant guidelines and regulations. The Institutional Human Ethics Committee of affiliated Baoan Hospital of Shenzhen, Southern Medical University approved the study protocols employed in this observational study. Written informed consent was obtained from each subject or their legal guardians.

\section{Consent for publication}

Not applicable.

\section{Availability of data and material}

The datasets used and analyzed during the current study are available from the corresponding author in response to reasonable requests.

\section{Competing interests}

The authors declare that they have no competing interests. 


\section{Funding}

This work was supported through funding from Wenwu Zhang (Shenzhen Key Medical Discipline Construction Fund, the Science and Technology Planning Project of Shenzhen Municipality, Grant Nos. JCY20140330093901761 and JCYJ20170307094345589), Jinle Lin (The Science and Technology Planning Project of Shenzhen Municipality, Grant Nos. JCYJ20180305123707368) and Jian Wu (National Natural Science Foundation of China, Grant Nos.81970012).

All of funders equally contribute to this investigation.

\section{Authors' contributions}

Conceived and designed the study: XF, JL. Study design Assessment: WZ, YX and WJ. Data acquisition: WT, JW, SZ, YL and YS. Blood collection and handling: XF, XZ and JY. Analyzed the data: JL, XF and SS. Wrote the paper: XF and JL. Provided critical appraisals of the study: XJ, QD, ZH and LW. Revised the manuscript: SS, WZ and YX. All authors read and approved the final manuscript.

\section{Acknowledgements}

We thank all the investigators for their excellent assistances in this clinical research.

\section{References}

1. Thompson BT, Chambers RC, Liu KD. Acute Respiratory Distress Syndrome. N Engl J Med. 
2017;377(6):562-572.

2. Matthay MA, Zemans RL, Zimmerman GA, et al. Acute respiratory distress syndrome. Nat Rev Dis Primers. 2019;5(1):18.

3. Force ADT, Ranieri VM, Rubenfeld GD, et al. Acute respiratory distress syndrome: the Berlin Definition. JAMA. 2012;307(23):2526-2533.

4. Rello J, Valenzuela-Sanchez F, Ruiz-Rodriguez M, Moyano S. Sepsis: A Review of Advances in Management. Adv Ther. 2017;34(11):2393-2411.

5. Noreen van der Linden, Karin Wildi RT, John W Pickering, Than M. Combining HighSensitivity Cardiac Troponin I and Cardiac Troponin $\mathrm{T}$ in the Early Diagnosis of Acute Myocardial Infarction. Circulation. 2018;138(10):989-999.

6. Fremont RD, Koyama T, Calfee CS, et al. Acute lung injury in patients with traumatic injuries: utility of a panel of biomarkers for diagnosis and pathogenesis. J Trauma. 2010;68(5):11211127.

7. Ware LB, Koyama T, Billheimer DD, et al. Prognostic and Pathogenetic Value of Combining Clinical and Biochemical Indices in Patients With Acute Lung Injury. Chest. 2010;137(2):288296.

8. Bellani G, Laffey JG, Pham T, et al. Epidemiology, Patterns of Care, and Mortality for Patients With Acute Respiratory Distress Syndrome in Intensive Care Units in 50 Countries. JAMA. 2016;315(8):788-800.

9. Ware LB, Calfee CS. Biomarkers of ARDS: what's new? Intensive Care Med. 2016;42(5):797799.

10. Lorraine B Ware, Tatsuki Koyama, Zhiguo Zhao, et al. Biomarkers of lung epithelial injury and 
inflammation distinguish severe sepsis patients with acute respiratory distress syndrome. Crit Care. 2013;17(5):R253.

11. Sapru A, Liu KD, Wiemels J, et al. Association of common genetic variation in the protein C pathway genes with clinical outcomes in acute respiratory distress syndrome. Crit Care. 2016;20(1):151.

12. Lin J, Zhang W, Wang L, Tian F. Diagnostic and prognostic values of Club cell protein 16 (CC16) in critical care patients with acute respiratory distress syndrome. J Clin Lab Anal. 2018;32(2).

13. Jiang Z, Zhou Q, Gu C, Li D, Zhu L. Depletion of circulating monocytes suppresses IL-17 and HMGB1 expression in mice with LPS-induced acute lung injury. Am J Physiol Lung Cell Mol Physiol. 2017;312(2):L231-L242.

14. Serpa Neto A, Campos PP, Hemmes SN, et al. Kinetics of plasma biomarkers of inflammation and lung injury in surgical patients with or without postoperative pulmonary complications. Eur J Anaesthesiol. 2017;34(4):229-238.

15. Agrawal A, Matthay MA, Kangelaris KN, et al. Plasma angiopoietin-2 predicts the onset of acute lung injury in critically ill patients. Am J Respir Crit Care Med. 2013;187(7):736-742.

16. Matthieu Jabaudon, Nacim Hamroun, Laurence Roszyk, et al. Effects of a recruitment maneuver on plasma levels of soluble RAGE in patients with diffuse acute respiratory distress syndrome: a prospective randomized crossover study. Intensive Care Med. 2015;41(5):846-855.

17. Park J, Pabon M, Choi AMK, et al. Plasma surfactant protein-D as a diagnostic biomarker for acute respiratory distress syndrome: validation in US and Korean cohorts. BMC Pulm Med. 2017;17(1):204.

18. Lin J, Shi M, Lu Y, et al. The changes of four cell-resource biomarkers in rats with acute 
respiratory distress syndrome. Chin J Emerg Med. 2020;29:1423-1430.

19. Lin J, Li J, Shu M, et al. The rCC16 Protein Protects Against LPS-Induced Cell Apoptosis and Inflammatory Responses in Human Lung Pneumocytes. Frontiers in Pharmacology. 2020;11.

20. Pang M, Liu HY, Li T, et al. Recombinant club cell protein 16 (CC16) ameliorates cigarette smokeinduced lung inflammation in a murine disease model of COPD. Mol Med Rep. 2018;18(2):2198-2206.

21. Zhou R, Yang X, Li X, et al. Recombinant CC16 inhibits NLRP3/caspase-1-induced pyroptosis through p38 MAPK and ERK signaling pathways in the brain of a neonatal rat model with sepsis. Journal of Neuroinflammation. 2019;16(1).

22. Schuldt EA, Lieb W, Dorr M, et al. Circulating angiopoietin-2 and its soluble receptor Tie-2 concentrations are related to inflammatory markers in the general population. Cytokine. 2018;105:1-7.

23. Dozio E, Ambrogi F, de Cal M, Vianello E, Ronco C, Corsi Romanelli MM. Role of the Soluble Receptor for Advanced Glycation End Products (sRAGE) as a Prognostic Factor for Mortality in Hemodialysis and Peritoneal Dialysis Patients. Mediators of Inflammation. 2018;2018:1-7.

24. Wannamethee SG, Welsh P, Papacosta O, et al. Circulating soluble receptor for advanced glycation end product: Cross-sectional associations with cardiac markers and subclinical vascular disease in older men with and without diabetes. Atherosclerosis. 2017;264:36-43.

25. Boschetto P, Campo I, Stendardo M, et al. Plasma sRAGE and N-(carboxymethyl) lysine in patients with CHF and/or COPD. Eur J Clin Invest. 2013;43(6):562-569.

26. Brodska H, Malickova K, Valenta J, Fabio A, Drabek T. Soluble receptor for advanced glycation end products predicts 28 -day mortality in critically ill patients with sepsis. Scandinavian 
Journal of Clinical and Laboratory Investigation. 2013;73(8):650-660.

27. Pregernig A, Muller M, Held U, Beck-Schimmer B. Prediction of mortality in adult patients with sepsis using six biomarkers: a systematic review and meta-analysis. Ann Intensive Care. 2019;9(1):125.

28. Anderson BJ, Calfee CS, Liu KD, et al. Plasma sTNFR1 and IL8 for prognostic enrichment in sepsis trials: a prospective cohort study. Critical Care. 2019;23(1).

29. Kaieda S, Gono T, Masui K, et al. Evaluation of usefulness in surfactant protein D as a predictor of mortality in myositis-associated interstitial lung disease. PLoS One. 2020;15(6):e0234523.

30. Wutzler S, Lehnert T, Laurer H, et al. Circulating levels of Clara cell protein 16 but not surfactant protein D identify and quantify lung damage in patients with multiple injuries. $J$ Trauma. 2011;71(2):E31-36.

31. Ye J, Jin L, Zhong C, et al. The clinical value of combined detection of serum angiopoietin 2 and Clara cell protein 16 in the early diagnosis of acute respiratory distress syndrome. Chin $J$ Emerg Med. 2019;28(9):1112-1117.

32. Spadaro S, Park M, Turrini C, et al. Biomarkers for Acute Respiratory Distress syndrome and prospects for personalised medicine. J Inflamm (Lond). 2019;16:1.

33. Binnie A, Tsang JL, dos Santos CC. Biomarkers in acute respiratory distress syndrome. Curr Opin Crit Care. 2014;20(1):47-55. 


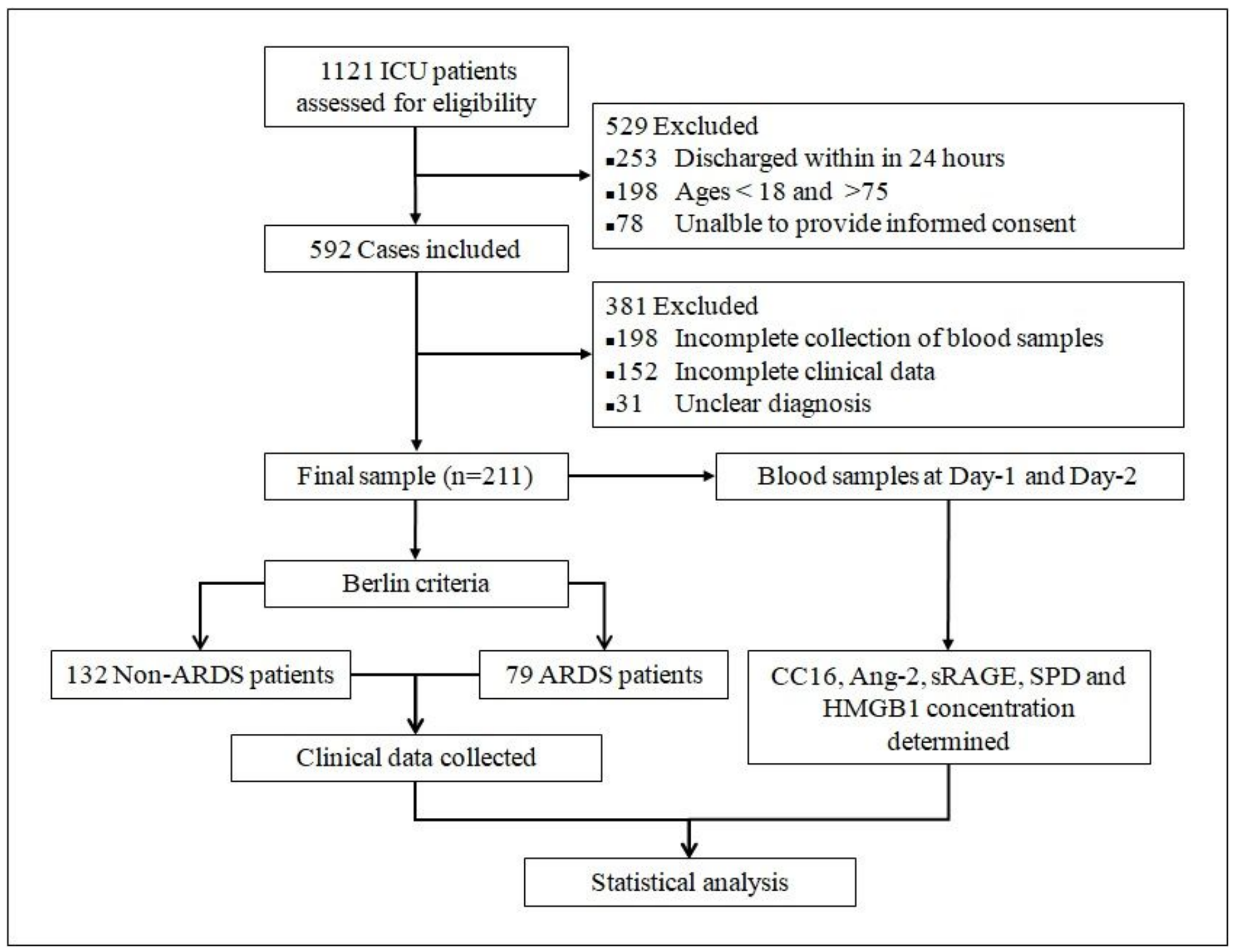

Figure 1

Flowchart of enrolment and study processes Reasons for exclusion were not mutually exclusive or exhaustive because there may have been a number of reasons for exclusion. Abbreviations: ICU, intensive care unit; ARDS, acute respiratory distress syndrome; CC16, club cell protein 16; Ang2, angiopoietin 2; sRAGE, soluble receptor for advanced glycation end-products; SPD, surfactant protein D; HMGB1, high mobility group box 1 

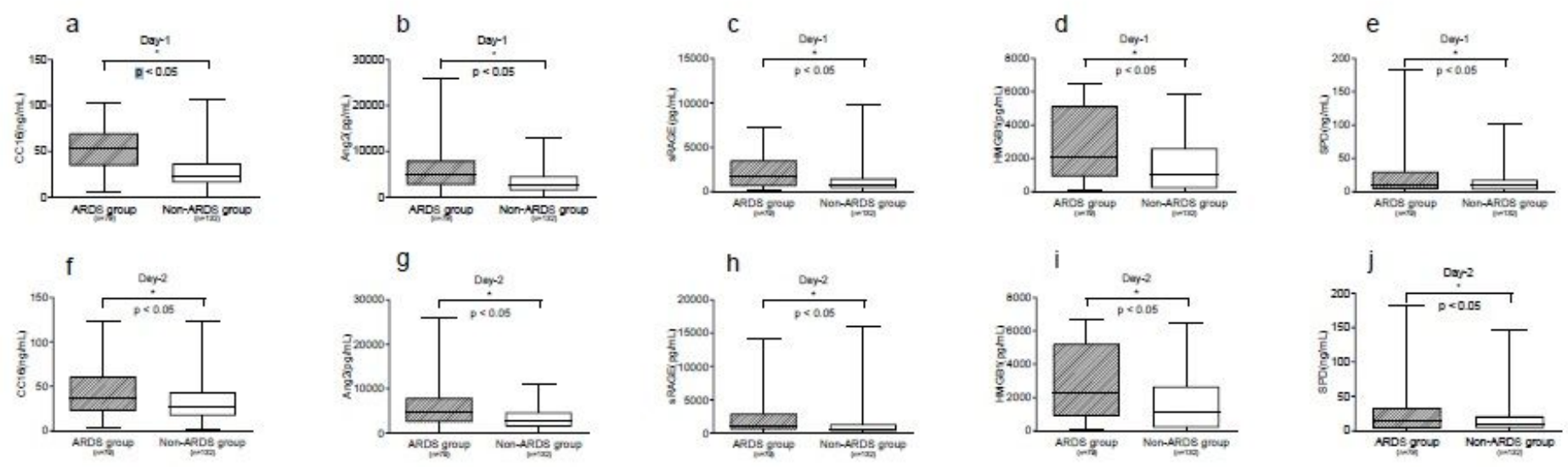

\section{Figure 2}

Bar graph for comparison of CC16, Ang-2, sRAGE, HMGB1 and SPD levels between groups. The $p$ values were derived from using student's t-test or Mann Whitney $\mathrm{U}$ test.
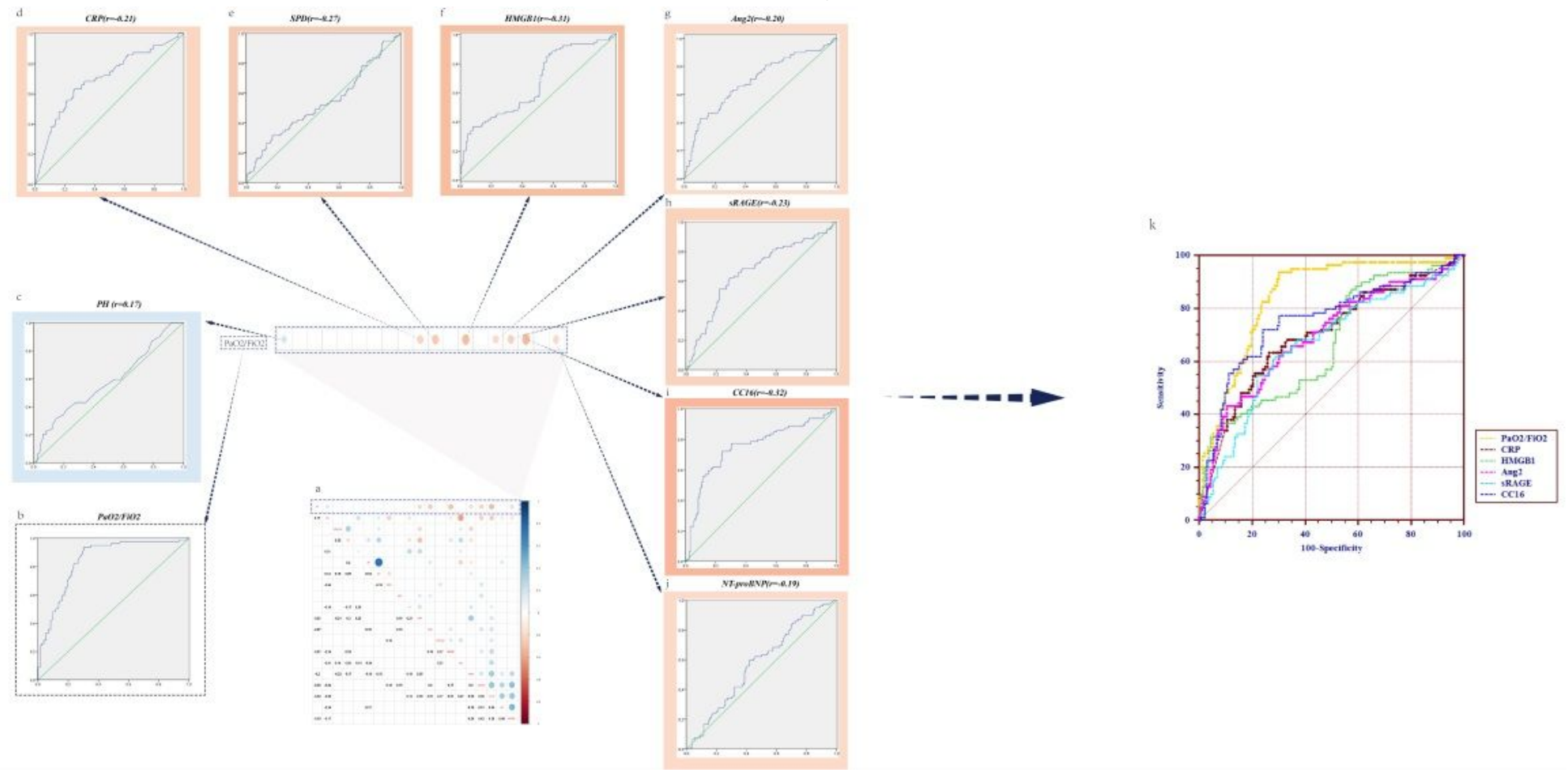

\section{Figure 3}

(a) Correlations between P/F, PH, Albumin, TP, T, SP, DP, WBC, RR, H, CRP, SPD, D-dimer, HMGB1, Lac, Ang2, sRAGE, CC16, Scr and NT-proBNP were investigated using Pearson's correlation coefficient. The ratio R was calculated by using Pearson's correlation coefficients ranging from -1 to 1 . Negative correlations have been highlighted in blue and positive correlations in red. Highlighted correlation coefficients indicate that correlations significantly differ from zero, and an emptyspace indicates the $r$ ratio had no significant difference. (b-j) ROC curves of P/F, PH, CRP, SPD, HMGB1, Ang-2, sRAGE, CC16 and NT-proBNP in diagnosing ICU patients with ARDS. The Pearson's correlation between $\mathrm{P} / \mathrm{F}$ and other indicators were 
referred to the $r$ value above. A negative value of $r$ indicated a negative correlation, or vice versa. $(k)$ ROC curves analysis showed the AUC of P/F, CRP, HMGB1, Ang-2, sRAGE and CC16, respectively. A logistic regression model was fit for diagnosis performance of ARDS using P/F, CRP and four biomarkers; the model performance was assessed by the AUC of the ROC. Abbreviations: P/F, PaO2/FiO2; TP, serum total protein; T, temperature; SP, systolic blood pressure; DP, diastolic blood pressure; WBC, white blood cell count; RR, respiratory rate; $\mathrm{H}$, heart rate; $\mathrm{CRP}$, c-reactive protein; Lac, lactic acid; $\mathrm{Scr}$, serum creatinine; CC16, club cell protein 16; Ang-2, angiopoietin 2; SRAGE, soluble receptor for advanced glycation endproducts; HMGB1, high-mobility group box 1 protein; SPD, surfactant Protein D; NT-proBNP, the N-terminal of the prohormone brain natriuretic peptide; ICU, intensive care unit; ARDS, acute respiratory distress syndrome; AUC, area under the curve; ROC, receiver operator characteristic.

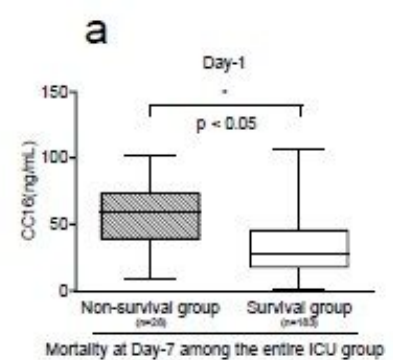

e
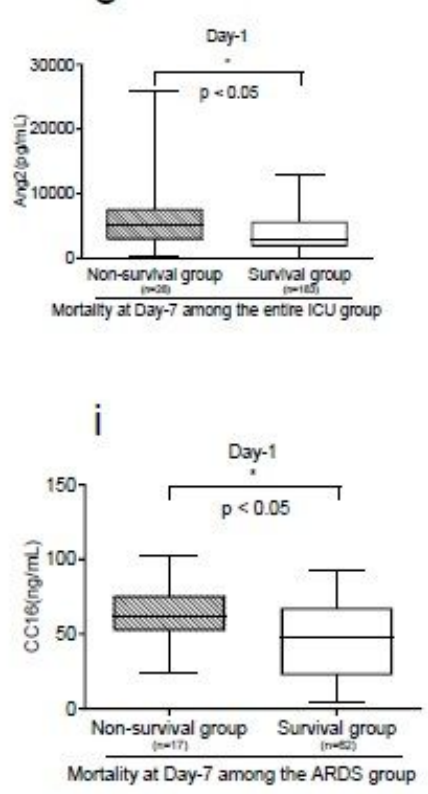

b

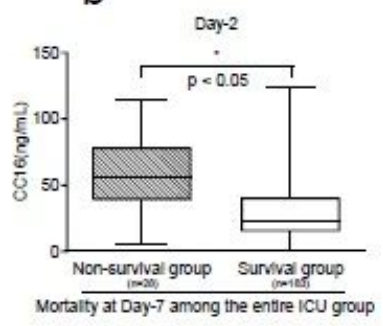

f
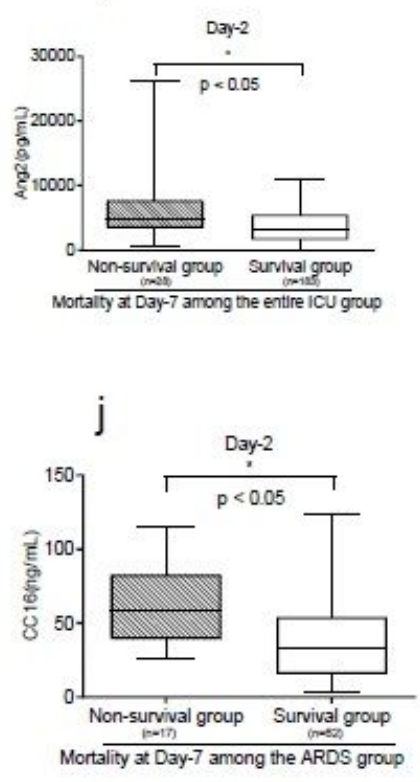

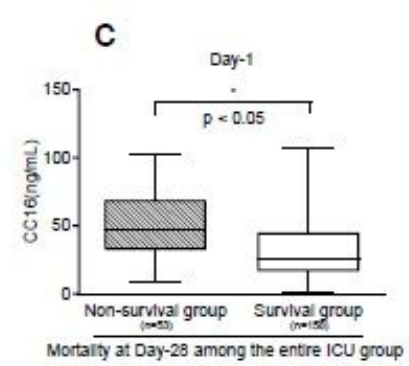

g
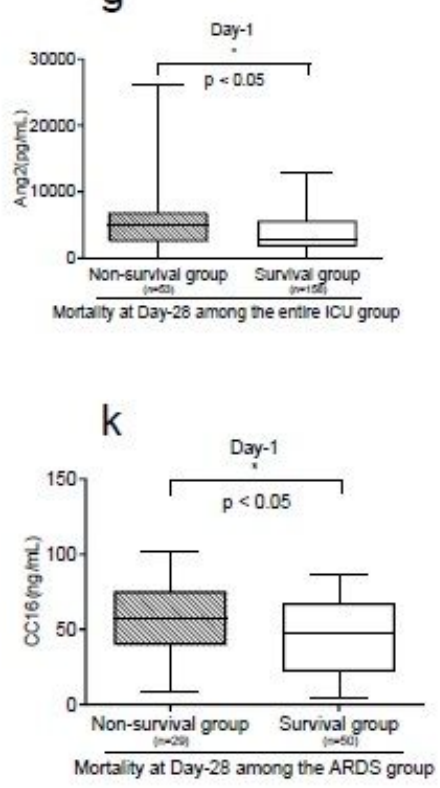

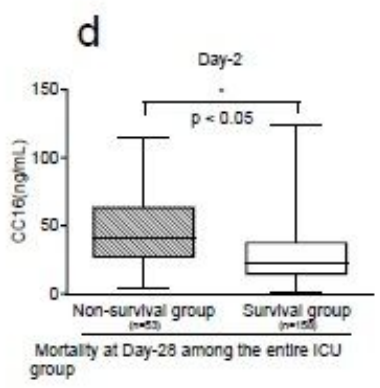

h
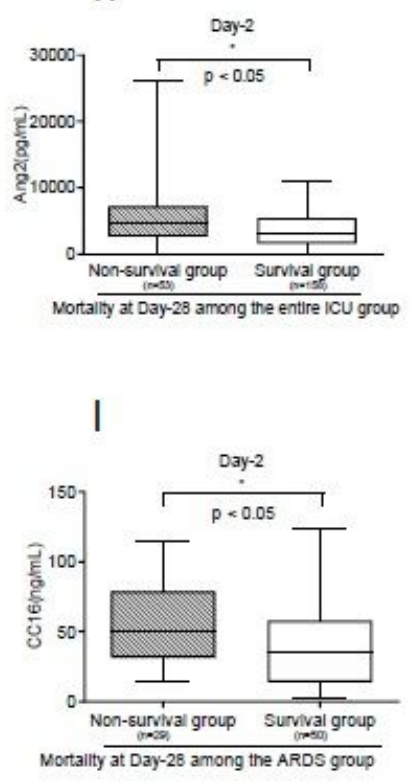

\section{Figure 4}

(a-h) Comparative bar charts of CC16 and Ang-2 between non-survival groups and survival groups across entire cohort. (i-j) Comparative bar charts of $\mathrm{CC} 16$ between non-survival groups and survival groups in ARDS only patients. $p$ values were generated using Student's T-test. 

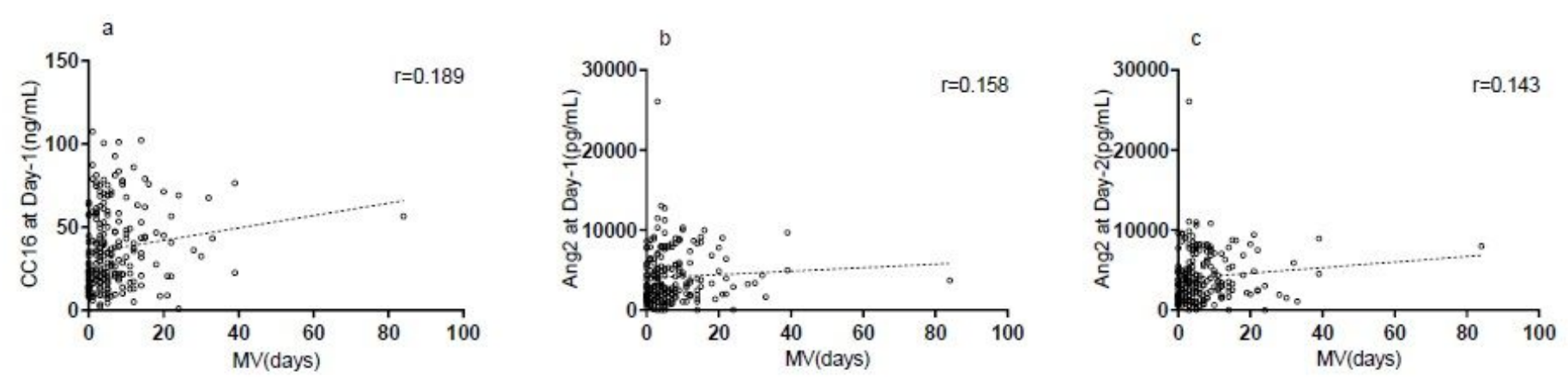

Figure 5

Scatter plots of a) the relationship between CC16 levels at Day-1 and duration of mechanical ventilation;

b) the relationship between Ang-2 levels at Day-1 and duration of mechanical ventilation; c) the relationship between Ang-2 levels at Day-2 and duration of mechanical ventilation. $r$ values were accounted from using Pearson correlation coefficient. Abbreviations: MV, mechanical ventilation duration; $r$, correlation coefficient

\section{Supplementary Files}

This is a list of supplementary files associated with this preprint. Click to download.

- AbbreviationList.docx 